\title{
L'OXYGÈNE DISSOUS SOUS LA GLACE
}

\author{
Par M. Paul VIVIER \\ Garde Général des Eaux et Forêts à Thonon-les-Bains (Haute-Savoie).
}

Il est intéressant de connaître, parmi les conditions physiques qui régissent la vie des poissons en hiver, la teneur en oxygène dissous des eaux closes, recouvertes de glace.

Cette question a son importance aussi bien pour les Salmonidés qui vivent dans les étangs et lacs de montagne, que pour les Cyprinidés des - langs et lacs de plaine, car si la Truite a besoin d'un minimum de 6 cc. d'oxygène par litre d'eau, la Carpe, moins exigeante, demande cependant pour vivre de 3 à $4 \mathrm{cc}$. de ce gaz.

Comme le principal facteur d'enrichissement de l'eau en oxygène est le contact de l'air, on pouvait se demander ce qu'il arrive lorsqu'une couche solide isole le milieu aquatique.

En Allemagne, où de nombreuses études ont été entreprises ces dernières années, principalement dans les eaux de la région de Plön, par le Professeur Thienemann (I), on a déjà recueilli des renseignements intéressants. Dans les lacs de moyenne profondeur, froids et longtemps recouvers de glace, comme le Pctit Ukleisee, le Plussee, la dose d'oxygène dissous est toujours faible en profondeur (fonds de i5 à 3o mètres) et parfois nulle ; en surface, la dose est très variable, allant de 30 à roo \% de saturation. Dans les lacs plus profonds, tels que le Grand lac de Plön, et qui gèlent irrégulièrement, la richesse du fond en oxygène, toujours suffisante, parait en rapport avec l'épaisseur de la glace, tandis que les couches superficielles et moyennes ne semblent pas subir de changements appréciables ; la surface est en général suffisamment oxygénée, atteignant mème souvent la dose de saturation.

Les phénomènes de sursaturation sont en effet assez fréquents l'hiver, dans les eaux gelées, mais très ensoleillées ; ainsi, dans le Grand Eutinersee, les couches d'eau superficielles se sont trouvées contenir jusqu'h I 5 cc. o8 d'oxygène par litre, soit $154.8 \%$ de saturation ; KNaUtue a rencontré le 2 I Janvier igir, dans une pièce d'eau, la dose prodigieuse de 46 cc. par litre (50o \% de saturation). Ce sont là, évidemment, des cas

(1) Thienemans (A). -.- Temperatur und Sauerstoff-Werhältnisse eisbedeckter Seen des Plöner Gebietes am linde des Winters : $9^{23-1934 . ~-~ A r c h . ~ f . ~ H y d r o b ., ~ X V I I I, ~}$ p. I, 1927 .

Halbfass (W.). - Wie geschieht die Anreicherung tiefer Wasserschichten von Seen und Meeren mit atmosphärischen Sauerstoff $\mathrm{P}$-.-Inlern. Verein. f. theore. $u$. angew. Limn., V, p. 284-297.

Thienemans (A.). - Der Sauersloff in eutrophen und oligotrophen Seen. - Die Binnengewasser, IV, Stuttgart $\mathrm{y}_{2} 8$. 
extrèmes et qu'il serait peut-être bon de vérifier. Ils peuvent s'expliquer par l'influence du plancton végétal qui, à la vive lumière, dégage une forte quantité d'oxygène. La circulation d'eau qui se fait sous la glace, dans certains lacs, a aussi un eaction enrichissante certaine

L'extrême pauvreté de certains fonds paraît liée tant à des phénomènes de décomposition qui vont jusqu'à produire de l'hydrogène sulfuré qu'à la faible assimilation chlorophylienne d'un plancton mal éclairé ( $\mathbf{I}$ ).

Les conditions de vie offertes aux Salmonidés en hiver, sous la glace, sont donc extrêmement variables suivant les lacs et, dans un même lac, suivant les jours (action du soleil) : ainsi l'Edebergsee, qui contenait à 5 mètres ro cc. 79 d'oxygène, n'avait plus à $8^{\mathrm{m}} 50$ que $\mathrm{I}$ cc. 4 et à 9 mètres, 5 cc. 32 dans deux autres observations ( 77 Mars I924, 4 Novembre igig).

Nous avons essayé de déterminer la teneur en oxygène, en hiver, sous la glace d'un lac et d'un étang de Savoie : - le lac de Montriond, l'étang de Marclaz.

Les dosages ont été effectués par la méthode WinkLer (2), la plus simple et la plus précise. Les prises d'échantillon ont été faites au moyen de la sonde Friedingen fermant hermétiquement et permettant de ramener à la surface une eau prélevée à la profondeur voulue; afin d'éviter tout contact avec l'oxygène de l'air, le contenu a été siphoné dans des flacons bouchés à l'émeri, l'oxygène a été fixé sur les lieux mêmes et aussitôt après la prise ; les solutions ont été faites à l'eau distillée et l'hyposulfite a été soigneusement purgé de gaz carbonique par une ébulition prolongée. La formule :

$$
\frac{n \times 0,0558 \times 1000}{\mathrm{~V}-\mathrm{A}}
$$

où $n$ est le nombre de cc. d'hyposulfite utilisé :

$V$, le volume du récipient contenant de l'eau à analyser ;

A, le volume de la solution basique d'iodure de potassium et de la solution de chlorure de manganèse utilisées ;

0,0558 , le volume d'oxygène correspondant à i cc. de la solution d'hyposulfite $\mathrm{N} /$ ıoo employée,

(I).Wasmund (E). - Lakustriche Interwasserböden. - Handbuch der Bodenlehre, par Blanck, I 930 .

(a) Hzbault (E.). - Contribution à l'étude des invertébrés torrenticoles. Paris 1927.

Vivier (P.). - Observations sur quelques facteurs hydrobiologiques piscicoles dans des lacs de Savoie. - Annales de l'Institut national agronomique, T. XXIV, r932.

Matcha (R.). - Hydrochemische Methoden in der Limnologie. - Die Binnengewas. ser, XII, Ig32.

Duparc (L.) et Basadonva (M.). - Manuel théorique et pratique d'analyse volumétrique, Genève Igı. 
: tonne le volume d'oxygène en centimètres cubes par litre d'eau à o ot à 76 centimètres de mercure.

Il faut noter que les rèsultats ne sont exacts qu'au dixième de centimètre cube. En effet, une erreur de lecture possible de $\mathrm{s} /$ io de cc. dans la solution d'hyposulfite donne, si $\mathrm{V}=256 \mathrm{cc}$, $\Lambda=6 \mathrm{cc}$. :

$$
\frac{0,1 \times 0,0558 \times 1000}{250}=0,02 \mathrm{cc} \text {. }
$$

Dans les résultats donnés nous n'avons conservé que le premier chiffre après la virgule qui, lui, ne peut être atteint par des erreurs de lecture.

Les températures ont été prises au thermomètre de marine tenu immergé 5 minutes.

$$
\text { I. - Le lac de Montriond (Haule-Savoie). }
$$

Ce lac d'éboulement, d'une superficie de 34 hectares, est situé à une altiture d'environ 1.050 mètres dans un diverticule de la vallée de la Dranse de Morzine. La profondeur est variable, par suite d'infiltrations souterraines dues à un colmatage incomplet, mais ne dépasse pas 16 mètres en période de pluie ou de fonte des neiges. Il n'a qu'un affluent et qu'un émissaire. Il contient des Truites communes (Salmo Iruttr L.) et des vairons (Phoxinus phioxinus L.).

\begin{tabular}{|c|c|c|c|c|c|c|}
\hline \multirow{2}{*}{ IIEUX DE PRISES D'ECH INTILILON } & \multicolumn{3}{|c|}{$\begin{array}{c}\text { Le } 8 \text { Février } 1932 \text { (1) } \\
\text { de } 15 \text { a } 16 \text { heures } \\
\text { Température de l'air : }-4^{\circ}\end{array}$} & \multicolumn{3}{|c|}{$\begin{array}{c}\text { Le'8 Mars } 1933(2) \\
\text { de } 15 \mathrm{~h} .1 / 2 \text { à } 16 \mathrm{~h} .1 / 2 \\
\text { Temperature de l'air : + } 1 .\end{array}$} \\
\hline & $\begin{array}{l}\text { Oxygène } \\
\text { en ce } \\
\text { par litre }\end{array}$ & $\begin{array}{l}\text { Tempé- } \\
\text { rature }\end{array}$ & $\begin{array}{l}\text { \% } \\
\text { desatu- } \\
\text { ration }\end{array}$ & $\begin{array}{c}\text { Oxygene } \\
\text { en cc. } \\
\text { par litre }\end{array}$ & $\begin{array}{l}\text { Tempé- } \\
\text { rature }\end{array}$ & de sutu- \\
\hline 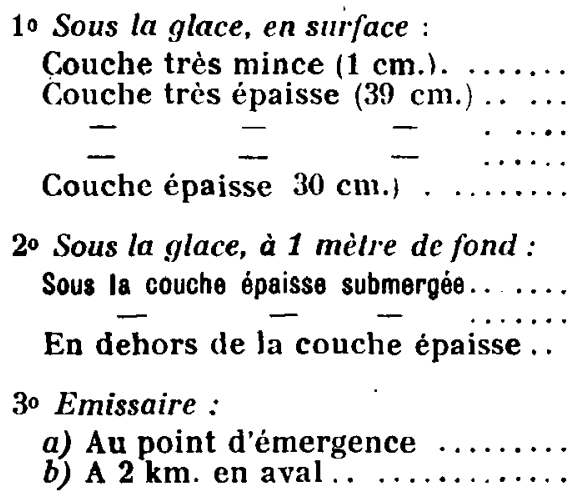 & $\begin{array}{l}6,7 \\
7,1 \\
6,7\end{array}$ & $\begin{array}{c}? \\
+3^{\circ}\end{array}$ & $\begin{array}{l}65 \% \\
69 \% \\
65 \%\end{array}$ & $\begin{array}{l}7,7 \\
8,4 \\
8,0\end{array}$ & +10 & $\begin{array}{c}? \\
\\
78 \% \\
85 \% \\
82 \%\end{array}$ \\
\hline $\begin{array}{l}\text { (1) Temps clair, mais par suite de l'expositi } \\
\text { (2) Temps neigeux. } \\
\text { (3) } 5 \text { minutes après, au mème point. } \\
\text { (4) } 15 \text { minutes après, au méme point, mais }\end{array}$ & légèr & es & leil. & 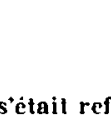 & 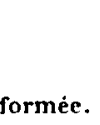 & \\
\hline
\end{tabular}

Des dosages ont été effectués alors que le lac était gelé (voir tableau) :

I - Le 8 Février 1932. - La couche de glace était de 39 centimètres. Malheureusement, par suite de difficultés d'accès, je n'ai pu emporter la 
sonde de Friedinger ni exécuter, par conséquent, de dosages en prolondeur.

1I. - Le 8 Mars 1933. -- Lne crue inattendue de l'affluent s'était produite la veille, avait soulevé l'épais manteau de glace et dislóqué celui-ci sur les bords ; il s'était reformé aussitòt une très mince pellicule solide, mais qui rendait impossible l'accès du milieu du lac et par conséquent les dosages, dans la plus grande profondeur ( 1 \% mètres environ).

$I^{\circ}$ Sous la glace, en surface, la tencur en oxygène est assez faible en $\mathrm{I}^{3} 32$; ̀̀ l'air, l'eau s'enrichit rapidement : $7 \mathrm{cc}$. I au lieu de $6 \mathrm{cc} .7$, au mème point, $\bar{j}$ minutes plus tard; le 8 Mars 1933 , par suite, sans doute, de l'apport d'eau oxygénée par la crue de la veille, les couches superfisielles sont plus riches : $8 \mathrm{cc} .4$; il est à remarquer que cette dose ets très voisine de celle observée le $\gamma$ Février I932, dans l'émissaire du lac (8 cc. oj), où l'eau était aérée.

$2^{\circ}$ Sous la glace, en profondeur, les résultats ne peuvent ètre concluants pür suile de l'affluence d'eau qui a rompu le 7 Mars ig33 l'équilibre du lac. Les teneurs varient de $7 \mathrm{cc}$. 7 à $8 \mathrm{cc}$. 4, un peu plus faibles, cependant qu'en surface ( $8 \mathrm{cc}$. en temps normal) ; elles doivent être inférieures, surtout dans de plus grands fonds, mais sans jamais atteindre des volumes vcisins de o ; il s'établit en effet à Montriond, un courant d'eau faible, mais continu de l'affluent à l'émissaire souterrain, qui doit apporter une certaine aération à l'eau close. Durant l'été, du reste, le lac n'a jamais en profondeur les faibles teneurs que l'on observe dans les lacs eutrophes ( $\mathrm{I}$ ).

$3^{\circ}$ L'émissaire, à son point d'émergence, n'a pas, ce qui est normal, une eau plus riche que le lac lui-mème : $6 \mathrm{cc}$. 7 , mais il ne tarde pas à s'oxygéniser au contact de l'air el 2 kilomètres plus bas, il contient déjà 8 cc. 05 , bien que de débit faible.

Si nous comparons ces résultats à ceux que nous avons obtenus dans te mème lac le 8 Août r 930 (2), nous constatons qu'en surface l'eau est un peu plus éloignée, l'hiver, de son degré de saturation en oxygène.

Dans un lac eutrophe, tel que l'Ukleisee comparable au lac de Montriond, Thenemann a trouvé à o mètre, le 18 Mars $1924,7 \mathrm{cc} .74$ et à I mètre, le 15 Novembre $1922,7 \mathrm{cc} .89$, volumes analogues aux nôtres ; ni dans le Plussee, ni dans le Cirand Ulileise, il n'a constaté normalement des phénomènes de sursaturation.

$$
\text { II. - Etang de Marclaz (Haute-Savoie). }
$$

Cet élang situé à environ 400 mètres d'altitude n'a qu'une superficie de quelques ares et sa profondeur ne dépasse pas $\mathrm{I}^{\mathrm{m}} 30$; alimenté et desservi par un petit ruisseau à cours rapide, il ne gèle pas complètement. Des íruites arc-en-ciel d'élevage se plaisent dans ses eaux.

(1) Vivien, op. cit., p. 15. - On appelle lacs eutrophes des lacs peu profonds, riches en ćlćments azotés, en humus et le plus souvent, en chaux. Ces lacs, très nombreux en montagnes, sont reconnaissables au ton vert ou bleu-vert de leurs eaux.

(a) Ibid., p. 47 . 
Le résultat des dosages est contenu dans le tableau suivant :

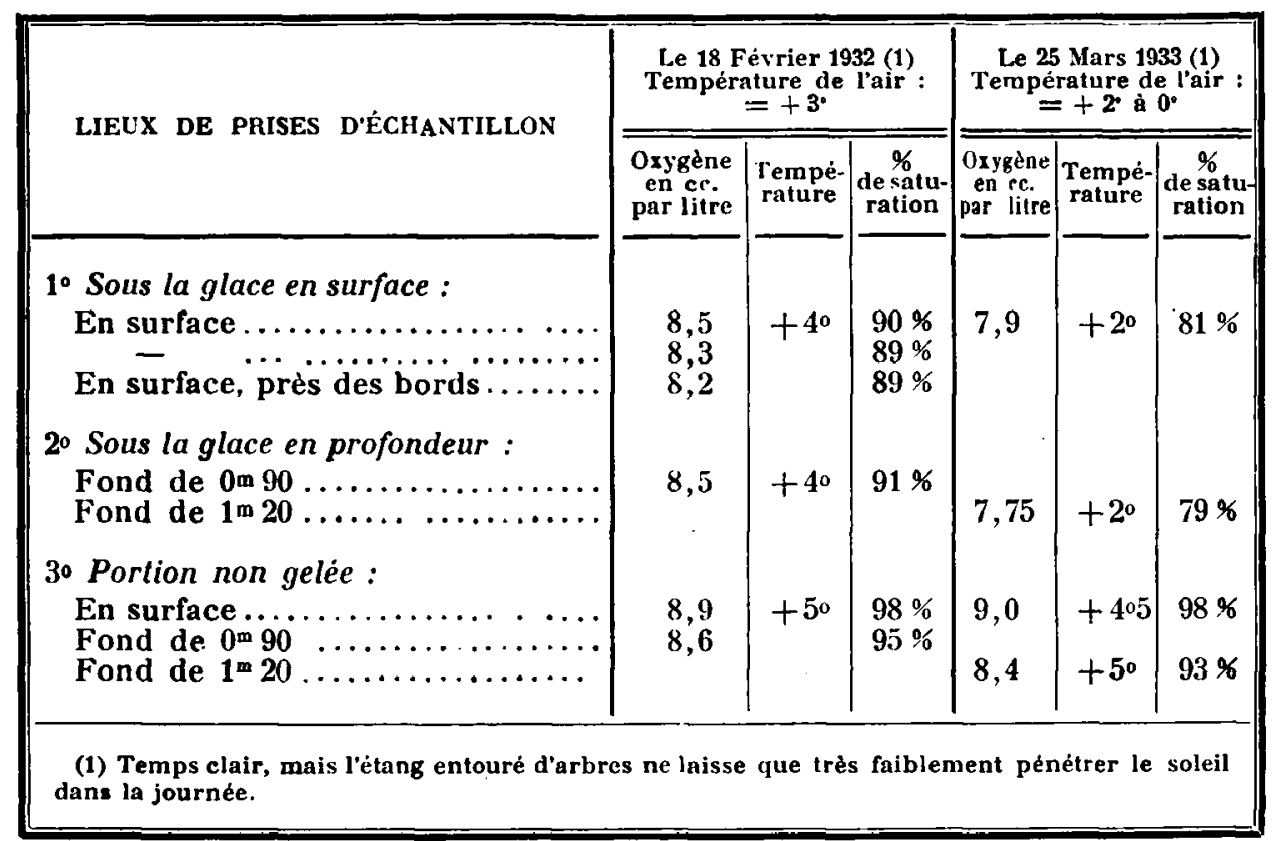

$I^{\circ}$ Sous la glace, en surface l'eau est suffisamment oxygénisée ( 8 cc. $3-8$ cc. 5 en $19^{3}, 7$ cc. 9 en r $\left.9^{33}\right)$. Près des rives, on note une légère diminution, très normale $(8 \mathrm{cc} .2)$. Si l'eau est moins riche en r 933 , sans doute faut-il en chercher la cause dans le plus grand développement de la couche de glace.

$2^{\circ}$ Sous la glace, en profondeur, il n'y a pas de diminution sensible : 7 cc. 75 en 1933,8 cc. 5 en I $_{9} 32$. L'étang est peu profond et il y a circulation d'eau.

$3^{\circ}$ En eau libre, en surface, à l'arrivée de l'affluent, la teneur du gaz dissous est beaucoup plus forte : 8 cc. 9 en $\mathrm{I}_{9} 3_{2}, 9$ cc. en $\mathrm{r}_{9} 33$.

En profondeur la teneur est moindre, mais supérieure cependant à celle qui s'observe sous la glace, à la même profondeur ( 8 cc. 6 en I 932 , 8 cc. 4 en rg33).

Il serait imprudent de tirer de ces quelques dosages effectués dans des eaux peu profondes et agitées, des conclusions d'ensemble. Néanmoins, ils montrent que si la glace est en général une cause sensible de diminution d'oxygène, elle offre cependant, même lorsqu'elle est très épaisse (Montriond I932), des conditions de vie suffisantes aux Salmonidés et a fortiori aux Cyprinidés, du moins dans les couches superficielles, les scules étudiées.

Nous remercions respectueusement M. l'Inspecteur principal des Eaux et Forêts Kneitmann, qui nous a suggééé ce travail, et M. l'Ingénieur en chef des Ponts et Chaussées Delebecque qui nous a encouragé dans l'étude de l'oxygène dissous. 